\title{
Acoustic Analysis of Intra-word Syllabic Timing Relations in Anterior Aphasia
}

\author{
Shari R. Baum \\ McGill University
}

\begin{abstract}
A study was conducted to explore intra-word syllabic timing relations in anterior, non-fluent aphasic patients. Ten sets of three words of increasing length (e.g. "sleep," "sleepy," sleepiness") were elicited from four non-fluent aphasic subjects. Acoustic analyses revealed that, overall, the aphasic patients produced monosyllabic root words with longer durations than those root words embedded in multisyllabic utterances. This general pattern was consistent with that for normal subjects reported in the literature (Lehiste 1972). However, the aphasic patients exhibited anomalous results for root word durations in the three-syllable condition. That is, root word durations were increased in the three-syllable compered to the two-syllable condition a pattern which contrasts with that of normal subjects. Results are discussed in relation to theories which attempt to characterize the speech production deficit in anterior aphasia.
\end{abstract}

Acoustic-phonetic research exploring the speech of aphasic patients has demonstrated that the speech production deficits exhibited by anterior (non-fluent) aphasics are likely due to impairments in articulatory implementation; in contrast, posterior (fluent) aphasics display deficits in phonological planning (e.g. Alajouanine et al. 1939; Blumstein et al. 1980; Blumstein et al. 1977; Kent and Rosenbek 1983). More specifically, studies have revealed that the anterior aphasics' deficit lies mainly in the temporal coordination of two independent articulators necessary for accurate production of voicing and nasality in consonants (Blumstein et al. 1977, 1980; Itoh et al. 1980; Itoh et al. 1979; Shewan et al. 1984; Tuller 1984), whereas those parameters which do not depend on temporal integration for their accurate production are unimpaired (e.g. production of place of articulation in stop consonants; Shinn and Blumstein 1983).

More recent studies have continued to explore the control of timing relations in the 
speech of aphasic patients in order to determine whether the temporal deficit is pervasive, affecting all aspects of timing, or whether the deficit is more specific, affecting only the integration of two independent articulators. For example, in addition to the deficits found with accurate production of voice onset time and nasality in consonants, investigations have shown that, despite the ability to maintain adequate phonological contrasts, anterior aphasic patients demonstrate aberrant patterns of vowel and consonant duration as compared to normal speakers (e.g. Duffy and Gawle 1984; Ryalls 1986; Baum et al. 1990). More research is needed to clarify the nature of the aphasics' control of timing relations and to determine how widespread the temporal deficit may be.

One aspect of timing which has not been explored in great detail in aphasia is the control of what might be called suprasegmental duration - that is, the temporal patterns of units larger than the speech sound segment (e.g. syllables, phrases or sentences). It is often reported that overall sentence durations are longer in anterior aphasics' speech production than in speech produced by normal subjects, and that these patients' speaking rate is decreased as compared to normal (e.g. Kent and McNeil 1987). Although much research has explored rate effects on segmental and syllabic production in normal subjects, few investigations have examined these effects in aphasic patients (cf. Kent and McNeil 1987). Since low-level segmental duration is thought to be impaired in anterior aphasia, it is important both for models attempting to explain the aphasic deficit and for improved remediation techniques to examine the extent to which higher-level temporal relations may influence the impairment in segmental timing.

One type of higher-level temporal relation which may affect segmental production is inter-syllabic timing. An interesting, and as yet unanswered, question is whether anterior aphasics demonstrate deviant patterns of inter-syllabic duration effects and, if so, to what extent these effects contribute to the perception of slowed speaking rate and to the segmental timing deficit attributed to these patients. In normal speech production, syllable (word) durations are longer when produced in isolation as compared to productions in multisyllabic contexts (Lehiste 1972). For example, a root morpheme such as "help" is longer in duration when produced in isolation than when the same morpheme is combined with additional affixes (e.g. "helpful," "helpfulness"). One hypothesis holds that this phenomenon is an example of the implementation of a rule of temporal control (e.g. Klatt 1976), an aspect of speech production which is impaired in anterior aphasia (Blumstein and Baum 1987). In other words, speakers may have learned a rule stating that one must decrease root syllable duration in multisyllabic words as a cue that additional syllables are upcoming; or conversely, the rule may indicate the need to lengthen utterance-final syllables as a cue to word and phrase segmentation. The implementation of temporal rules has been consistently shown to be impaired in anterior aphasia with reference to the production of voicing 
in stops and fricatives, the production of nasal consonants, and the production of vowels (see Blumstein and Baum 1987 for a review). Another potential explanation for the shortening phenomenon implicates coarticulation in speech production, that is, one aspect of coarticulation in normal speech production is the effect of final consonants on the duration of a preceding stressed vowel. Studies have shown that stressed vowel duration will decrease due to the addition of a final consonant or unstressed syllable (Lindblom and Rapp 1973). This effect has been attributed to the overlay of consonant production on tonic vowel production (see Fowler 1979). While a detailed explanation of Fowler's theory of coproduction is beyond the scope of this paper, a brief description is in order. Fowler's theory proposes that speech production is based on a stressed vowel to stressed vowel unit of production with consonantal and unstressed vowels coproduced or overlaid on this fundamental unit. That is, cues to both consonants and unstressed vowels are integrated over the basic unit. It follows, therefore, that if the base pattern remains stable, any additions to the pattern would shorten the duration of at least one of the stressed vowels. Although studies of this sort of coarticulation have not been reported for aphasic patients (but cf. Collins et al. 1983), other aspects of coarticulation have been shown to be impaired - notably, anticipatory labial coarticulation or the effect of an upcoming vowel on consonant production (e.g. Katz 1987; Tuller and Seider-Story 1987). Whatever the correct explanation for the syllable shortening phenomenon, it is important to determine whether the temporal deficit which has been attributed to anterior aphasics affects this suprasegmental level of durational control.

The goal of the present investigation was thus to provide a preliminary exploration of the phenomenon of syllabic shortening in multisyllabic utterances in anterior aphasic patients. If the aphasic patients demonstrate durational patterns different from the norm, it would suggest that the temporal deficit attributed to these subjects is not limited to the integration of independent articulators, but affects more 'global' temporal control as well. In contrast, should the anterior aphasic patients exhibit the normal pattern of syllabic shortening in words of increasing length, the results would indicate a spared capacity to plan and implement suprasegmental timing relations.

\section{METHODS}

\section{Subjects}

Four non-fluent aphasic patients served as subjects. Each was classified as having Broca's aphasia or apraxia of speech on the basis of clinical examination including both formal and informal tests (e.g. Boston Diagnostic Aphasia Examination (Goodglass and Kaplan 1983), Minnesota Test for the Differential Diagnosis of Aphasia (Schuell 1965)). Biographic data are presented in Table 1. (Unfortunately, 
specific site of lesion data are unavailable at this time.) All four subjects were native speakers of English.

\section{TABLE 1}

Profles of Aphasic Subjects Tested

\begin{tabular}{cccc}
\hline Subject & Age (years) & Diagnosis & Months Post Onset \\
\hline A1 & 33 & non-fluent, apraxic & 22 \\
A2 & 62 & agrammatic, apraxic & 14 \\
A3 & 40 & agrammatic, apraxic & 11 \\
A4 & 40 & non-fluent, apraxic & 6 \\
\hline
\end{tabular}

\section{Stimuli and Procedure}

The stimuli consisted of 10 sets of three words each: the root word (e.g. "sleep"), a two-syllable word including the root word (e.g. "sleepy"), and a three-syllable word including the root (e.g. "sleepiness"). Each word was printed on a $3 \times 5$ card and presented to the subjects in random order in the context of the phrase "Please say -." The entire set of stimuli is provided in Table 2 . The series of 30 randomized stimuli was elicited four times for a total of 120 stimuli per subject. If the subject

\section{TABLE 2}

Stimuli Sets Used in the Present Study

\begin{tabular}{lll}
\hline sleep & sleepy & sleepiness \\
self & selfish & selfishness \\
help & helpful & helpfulness \\
nose & nosy & nosiness \\
mud & muddy & muddiness \\
speed & speedy & speediness \\
blood & bloody & bloodiness \\
luck & lucky & luckiness \\
play & playful & playfulness \\
sheep & sheepish & sheepishness \\
\hline
\end{tabular}


was unable to read a stimulus, the token was produced by the examiner and repeated by the aphasic patient. As expected, the repetition procedure was more often required for the three-syllable stimuli.

The utterances were recorded in a quiet room with a Sony Professional WM-D3 cassette recorder and high-quality microphone. Stimuli were digitized via an ATcompatible computer using the BLISS speech analysis system (Mertus 1989) and edited from the waveform display. Tokens were sampled at a rate of $20 \mathrm{k}$ samples per second with an $8.5 \mathrm{kHz}$ low-pass filter and 12-bit quantization.

\section{Duration Analyses}

Durations were measured in all cases by both visual inspection of the acoustic waveform and auditory perception. For the root word tokens, durations were measured from the onset of initial consonant noise or nasal murmur (for $[\mathrm{m} \mathrm{n}]$ ) through the burst or frication noise associated with the word-final consonant. (All final stops were released by all subjects.)

For the polysyllabic words, durations were measured in a similar manner. Syllable onset was defined as above, while syllable offset was demarcated by the onset of periodic energy corresponding to the upcoming vowel and the end of frication or aspiration noise for the syllable-final consonant.

Tokens containing segmental omission or paraphasic error in the root portion of the stimulus were excluded from the analyses. A total of 33 (less than 7\%) of the stimuli was eliminated due to errors of this nature. Production errors in the second or third syllable of multisyllabic words were permitted as long as the integrity of the syllable size was maintained. (There was no test to ensure that the subjects understood the stimuli which they read since comprehension was not of concern to the specific analyses undertaken.)

\section{RESULTS}

Mean syllable durations and standard deviations (in ms) were calculated for each subject for each word triplet. These values collapsed across stimuli are presented in Table 3, along with mean values extrapolated from Lehiste (1972) for normal subjects. Overall means reflected well the data for individual word triplets. A cursory examination of the table reveals that all aphasic subjects produced monosyllables in isolation which were longer in duration than those same syllables embedded in multisyllabic words, with means across subjects of $542 \mathrm{~ms}$ in the monosyllable condition, $431 \mathrm{~ms}$ in the two-syllable condition, and $459 \mathrm{~ms}$ in the three-syllable condition. The mean difference between the monosyllabic and bisyllabic words across subjects was $111 \mathrm{~ms}$. This value corresponds well to a mean difference value extrapolated from 
TABLE 3

Mean Root Morpheme Durations in ms for Each Subject

\begin{tabular}{lccc}
\hline Subject & one-syllable & two-syllable & three-syllable \\
\hline A1 & $528(93)$ & $385(95)$ & $348(82)$ \\
A2 & $524(124)$ & $420(103)$ & $487(140)$ \\
A3 & $591(121)$ & $476(126)$ & $527(106)$ \\
A4 & $525(95)$ & $444(85)$ & $473(88)$ \\
\hline X & $542(33)$ & $431(38)$ & $459(77)$ \\
N (x across two & & & \\
$\quad$ subjects) & $460(57)$ & $345(27)$ & $287(24)$ \\
(extrapolated from Lehiste 1972) & & \\
\hline
\end{tabular}

Lehiste's (1972) data of $115 \mathrm{~ms}$. The difference in pattern which may be indicative of a significant difference between the aphasic patients and normals is revealed in the analyses of the three-syllable words. As is evident from the table, all but one of the anterior aphasic patients demonstrated increased root syllable duration in the threesyllable as compared to the two-syllable words. These results stand in sharp contrast to the decreased root syllable duration for three-syllable words reported by Lehiste (1972). The mean difference between root morpheme durations for two- and threesyllable words extrapolated from Lehiste's data is $58 \mathrm{~ms}$ (- a value not unlike that found for subject Al (37 ms)).

To explore this difference a bit further, ratios of the monosyllabic productions relative to the multisyllabic productions of the root syllable were calculated (see Lehiste 1972). Any value greater than 1.0 indicates a reduction in duration in the longer multisyllabic words; the larger the ratio, the greater the reduction. The ratio results are depicted graphically in Fig. 1 which displays the mean monosyllabic/ multisyllabic root duration ratios in the three syllabic conditions for each subject. (All ratios are equal to 1.0 in the one-syllable condition.) In no case did a monosyllable/ multisyllable ratio of less than 1.0 result. The figure reveals a very similar pattern for three of the four aphasic subjects, with ratios in the two-syllable condition much larger than in the three-syllable condition. In contrast, the ratios reported by Lehiste (1972) for normal subjects (plotted in Fig. 1) were substantially greater for the three-syllable as compared to the two-syllable conditions, with mean ratios (derived from her report) of 1.60 and 1.33, respectively. As may be seen, subject A1 displayed the pattern shown for normal subjects, with a larger ratio in the three-syllable than in the twosyllable condition. 


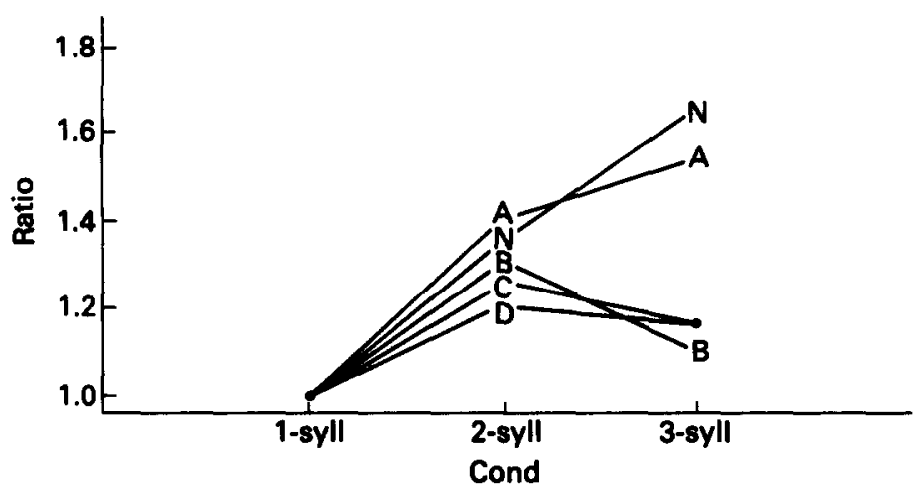

Figure 1. Ratios of Monosyllabic to Multisyllabic Root Morpheme Durations for each Subject. Subjects A1-A4 are Represented by the Letters A-D; The Normal Data Extrapolated from Lehiste (1972) are Represented by $\mathbf{N}$. A Solid Dot Indicates Overlapping Values.

\section{DISCUSSION}

The results of this preliminary investigation revealed that anterior, non-fluent aphasic patients consistently decrease the duration of a syllable when it is produced in a multisyllabic context as compared to when it is produced in isolation. This general finding is consistent with the durational pattern found in normal subjects in earlier reports (Lehiste 1972; Tye-Murray and Woodworth 1989).

In contrast to the normal pattern, three of the four aphasic patients demonstrated increases in duration in the three-syllable as compared to the two-syllable condition. This result may be attributed to the overall difficulty which anterior patients experience with multisyllabic word production, or the production of long stretches of speech, yielding a typical non-fluent character to their speech. That is, when faced with a long string to produce, patients may break it down into manageable length units and produce them "separately." This concept has been explored and discussed in detail with regard to apraxia of speech (Kent and Rosenbek 1982, 1983). Kent and Rosenbek $(1982,1983)$ have proposed three types of syllabic breakdown to account in part for the typically slowed rate of apraxic speakers: "syllable segregation," "syllable dissociation," and "articulatory prolongation." Syllable segregation is characterized by the temporal separation of syllables within a prosodic unit, i.e. increased silent intervals between syllables. Similarly, syllable dissociation is characterized by the isolation of syllables in the temporal domain, but it represents a more 
extreme separation in that no unifying prosodic contour is present across the syllable series. The results of the current study best approximate Kent and Rosenbek's notion of "articulatory prolongation," defined as the lengthening of components of a continuous speech stream. That is, the patients in the present study did not insert significant pauses between syllables in the three-syllable condition; rather, they increased intra-syllabic duration. Thus the hypothesis that subjects break down multisyllabic units and produce them separately is called into question. An alternative explanation is that the implementation of global timing relations necessary to produce the normal duration patterns with multisyllabic words breaks down with utterances of more than two syllables. Some evidence in support of this contention is provided by Kent and Rosenbek (1983) who state that "excessive lengthening" is more common in longer, multisyllabic utterances. In addition, breakdowns in the normal patterning of intensity relations across syllables have been demonstrated in multisyllabic utterances (Kent and Rosenbek 1983). This hypothesis of breakdown in words of more than two syllables remains speculative, though, and additional data are needed to substantiate or refute such a possibility.

A comment should be made on the different pattern of results for subject Al. This subject did not show an increase in duration for root morphemes in the three-syllable condition. In fact, she demonstrated root morpheme durations which were even further decreased in the three-syllable as compared to the two-syllable condition - the pattern reported for normal subjects. The discrepancy is likely attributable to the fact that subject $\mathbf{A l}$ had a considerably less severe deficit according to clinical records. Further, the longest amount of time had passed since her CVA (22 months) and she was the youngest subject in the group, factors which are thought to influence recovery of function in aphasia (Darley 1982). Another possibility relates to precise localization and extent of lesion. Unfortunately, we do not currently possess sufficient information to address this hypothesis (but see Baum et al. 1990).

Due to their preliminary nature, the current results are inconclusive with regard to determining the degree to which global temporal parameters are implicated subsequent to anterior brain damage. The data are suggestive, however, of a potential deficit in the control of timing of multisyllabic utterances. It is not clear whether this deficit lies at the level of programming of temporal relations or in their implementation. Given the results of previous studies, however, as well as the current study's finding that anterior patients correctly program and implement multisyllabic timing relations involving two-syllable words, it is likely that the temporal deficit demonstrated implicates the level of implementation.

The present investigation is part of a larger series of studies exploring the effects of speaking rate on the production of speech sound segments in aphasia. Any conclusive claims regarding the neuroanatomical bases of temporal aspects of speech motor control or the precise nature of the speech production deficit in anterior, non- 
fluent (as compared to posterior, fluent) aphasia must await results of the ongoing projects.

\section{REFERENCES}

Alajouanine, T., A. Ombredane and M. Durand

1939 Le syndrome de la desintegration phonetique dans l'aphasie, Paris: Masson.

Baum, S., S. Blumstein, M. Naeser and C. Palumbo

1990 "Temporal Dimensions of Consonant and Vowel Production: An Acoustic and CT Scan Analysis of Aphasic Speech," Brain and Language 39. $33-56$.

Blumstein, S. and S. Baum

1987 "Consonant Production Deficits in Aphasia," in Phonetic Approaches to Speech Production in Aphasia and Related Disorders, J. Ryalls (ed.), MA: College-Hill Press.

Blumstein, S., W. Cooper, H. Goodglass, S. Statlender and J. Gottlieb

1980 "Production Deficits in Aphasia: A Voice-onset Time Analysis," Brain and Language 9. 153-70.

Blumstein, S., W. Cooper, E. Zurif and A. Caramazza

1977 "The Perception and Production of Voice-onset Time in Aphasia," Neuropsychologia 15. 371-83.

Collins, M., J. Rosenbek and R. Wertz

1983 "Spectrographic Analysis of Vowel and Word Duration in Apraxia of Speech," Journal of Speech and Hearing Research 26. 224-30.

Darley, F.

1982 Aphasia, PA: W.B. Saunders Co.

Duffy, J. and C. Gawle

1984 "Apraxic Speakers' Vowel Duration in Consonant-vowel-consonant Syllables," in Apraxia of Speech, J. Rosenbek, M. McNeil and A. Aronson (eds), CA: College-Hill Press.

Fowler, C.

1979 "Perceptual Centers in Speech Production and Perception," Perception and Psychophysics 25. 375-88.

Goodglass, H. and E. Kaplan

1983 The Assessment of Aphasia and Related Disorders, PA: Lea and Febiger.

Itoh, M., S. Sasanuma, H. Hirose, H. Yoshioka and T. Ushijima

1980 "Abnormal Articulatory Dynamics in a Patient with Apraxia of Speech," Brain and Language 11. 66-75. 
Itoh, M., S. Sasanuma and T. Ushijima

1979 "Velar Movements During Speech in a Patient with Apraxia of Speech," Brain and Language 7. 227-39.

Katz, W.

1987 "Anticipatory Labial and Lingual Coarticulation in Aphasia," in Phonetic Approaches to Speech Production in Aphasia and Related Disorders, J. Ryalls (ed.), MA: College-Hill Press.

Kent, R. and M. McNeil

1987 "Relative Timing of Sentence Repetition in Apraxia of Speech and Conduction Aphasia," in Phonetic Approaches to Speech Production in Aphasia and Related Disorders, J. Ryalls (ed.), MA: College-Hill Press.

Kent, R. and J. Rosenbek

1982 "Prosodic Disturbance and Neurologic Lesion," Brain and Language 15. 259-91.

Kent, R. and J. Rosenbek

1983 "Acoustic Patterns of Apraxia of Speech," Journal of Speech and Hearing Research 26. $231-48$.

Klatt, D.

1976 "Linguistic uses of Segmental Duration in English: Acoustic and Perceptual Evidence," Journal of the Acoustic Society of America 59. 1208-21.

Lehiste, I.

1972 "The Timing of Utterances and Linguistic Boundaries," Journal of the Acoustic Society of America 51. 2018-24.

Lindblom, B. and K. Rapp

1973 "Some Temporal Regularities of Spoken Swedish," Paper of the Linguistic University of Stockholm 21. 1-59.

Mertus, J.

1989 BLISS, Brown University, Providence, RI.

Ryalls, J.

1986 "An Acoustic Study of Vowel Production in Aphasia," Brain and Language 29. $48-87$.

Schuell, $\mathbf{H}$.

1965 Differential Diagnosis of Aphasia with the Minnesota Test, MN: University of Minnesota Press.

Shewan, C., H. Leeper and J. Booth

1984 "An Analysis of Voice Onset Time (VOT) in Aphasic and Normal Subjects," in Apraxia of Speech, J. Rosenbek, M. McNeil and A. Aronson (eds), CA: College-Hill Press.

Shinn, P. and S. Blumstein

1983 "Phonetic Disintegration in Aphasia: Acoustic Analyses of Spectral 
Characteristics for Place of Articulation," Brain and Language 20. 90-114.

Tuller, B.

1984 "On Categorizing Aphasic Speech Errors," Neuropsychologia 22. 547-57. Tuller, B. and R. Seider-Story

1987 "Anticipatory Coarticulation in Aphasia," in Phonetic Approaches to Speech Production in Aphasia and Related Disorders, J. Ryalls (ed.), MA: College-Hill Press.

Tye-Murray, N. and G. Woodworth

1989 "The Influence of Final-syllable Position on the Vowel and Word Duration of Deaf Talkers," Journal of the Acoustic Society of America 85. 313-21. 